\title{
Alkaline phosphatase bone isoform B1x - a marker of impaired osteoblastic function in patients with renal osteodystrophy
}

\author{
Mathias Haarhaus a,b, Marie-Claude Monier-Faugerec, Per Magnussona* , Hartmut H. Malluche ${ }^{c *}$ \\ a Division of Clinical Chemistry, Department of Clinical and Experimental Medicine, Faculty of \\ Health Sciences, Linköping University, Linköping, Sweden \\ b Department of Nephrology, Karolinska University Hospital, Stockholm, Sweden \\ c Division of Nephrology, Bone and Mineral Metabolism, University of Kentucky, Albert B. \\ Chandler Medical Center, Lexington, USA \\ * These authors contributed equally as senior authors.
}

\section{Introduction}

Renal osteodystrophy encompasses the bone histologic abnormalities seen in patients with chronic kidney disease (CKD). The bone-specific alkaline phosphatase (BALP) isoform B1x is exclusively found in serum of some CKD patients. The aim of this study was to examine the relationship between serum BALP isoform activities and histomorphometric parameters of bone in patients with CKD on chronic dialysis.

\section{Methods}

Anterior iliac crest bone biopsy samples from 40 CKD patients were selected on the basis of bone turnover for histomorphometric analysis. There were samples from 20 patients with low and 20 with non-low bone turnover. In serum, BALP, BALP isoforms (B/I, B1X, B1 and B2), and parathyroid hormone (PTH) were measured.

\begin{tabular}{|c|c|c|}
\hline \multicolumn{3}{|c|}{$\begin{array}{l}\text { Bone histomorphometric parameters in patients on hemodialysis with } \\
\text { absence or presence of the circulating BALP isoform B1X }\end{array}$} \\
\hline & $\begin{array}{l}\text { B1x absent } \\
(n=19)\end{array}$ & $\begin{array}{l}\text { B1x present } \\
\quad(n=21)\end{array}$ \\
\hline \multicolumn{3}{|l|}{ Parameters of bone structure } \\
\hline Bone volume/Tissue volume (\%) & $20.1(16.5-23.1)$ & $19.8(15.8-24.7)$ \\
\hline Trabecular thickness $(\mu \mathrm{m})$ & $109(74.7-120)$ & $93.9(79.7-110)$ \\
\hline Trabecular separation $(\mu \mathrm{m})$ & $342(295-546)$ & $375(305-475)$ \\
\hline \multicolumn{3}{|l|}{ Static parameters of bone formation } \\
\hline Osteoid volume/Bone volume (\%) & $8.25(5.20-20.8)$ & $6.44(3.95-13.0)$ \\
\hline Osteoid surface/Bone surface (\%) & $43.8(24.6-54.6)$ & $35.2(15.7-46.5)$ \\
\hline Osteoid thickness $(\mu \mathrm{m})$ & $13.1(9.79-15.1)$ & $10.2(8.28-14.6)$ \\
\hline $\begin{array}{l}\text { Number of osteoblast/bone perimeter } \\
(\# / 100 \mathrm{~mm})\end{array}$ & $178(61.5-349)$ & $33.5(10.5-136)^{\#}$ \\
\hline \multicolumn{3}{|l|}{ Parameters of bone resorption } \\
\hline Erosion surface/Bone surface (\%) & $2.44(1.56-6.15)$ & $0.98(0.55-3.01)$ \\
\hline Erosion depth $(\mu \mathrm{m})$ & $13.0(11.8-16.4)$ & $11.0(9.04-13.8)$ \\
\hline $\begin{array}{l}\text { Number of osteoclast/Bone perimeter } \\
(\# / 100 \mathrm{~mm})\end{array}$ & $23.8(14.3-80.7)$ & $12.9(10.8-30.7)$ \\
\hline \multicolumn{3}{|l|}{ Dynamic parameters } \\
\hline Mineral apposition rate $(\mu \mathrm{m} / \mathrm{d})$ & $0.83(0.75-0.93)$ & $0.52(0.10-0.88)^{*}$ \\
\hline Mineralizing surface/Bone surface (\%) & $6.52(4.32-12.6)$ & $3.17(0.80-8.7)$ * \\
\hline $\begin{array}{l}\text { Bone formation rate/Bone surface } \\
\left(\mathrm{mm}^{3} / \mathrm{cm}^{2} / \mathrm{yr}\right)\end{array}$ & $2.43(1.57-3.86)$ & $0.55(0.03-3.04)$ * \\
\hline Activation frequency $\left(\mathrm{yr}^{-1}\right)$ & $0.57(0.33-0.65)$ & $0.16(0.01-0.65)$ * \\
\hline Mineralization lag time (days) & $70.1(46.1-136)$ & $225(90.1-972) \#$ \\
\hline $\begin{array}{l}\text { Bone formation rate/osteoblast } \\
\left(\mathrm{mm}^{3} / \mathrm{cell} / \mathrm{yr}^{*} 10^{3}\right)\end{array}$ & $15.3(5.07-38.5)$ & $9.97(2.59-21.6)$ \\
\hline Osteoblast vigor (\%/day) & $0.49(0.23-0.62)$ & $0.14(0.02-0.37)$ * \\
\hline
\end{tabular}

Conflict of interest statement

The authors have no conflict of interest to disclose.

\section{Results}

B1x was found in 21 patients (53\%). They had lower levels of BALP, other BALP isoforms, and PTH compared to patients without B1x. B1x correlated inversely with osteoblast number and activity. ROC curves showed that B1x was superior to PTH and BALP to diagnose low osteoblast activity.

\section{Conclusion}

The study shows that B1x is a novel diagnostic parameter for osteoblastic insufficiency as illustrated by superior ROC curve AUC compared toPTH and BALP. This indicates that the release of B1x into serum from bone is a sign of perturbed osteoblast activity.

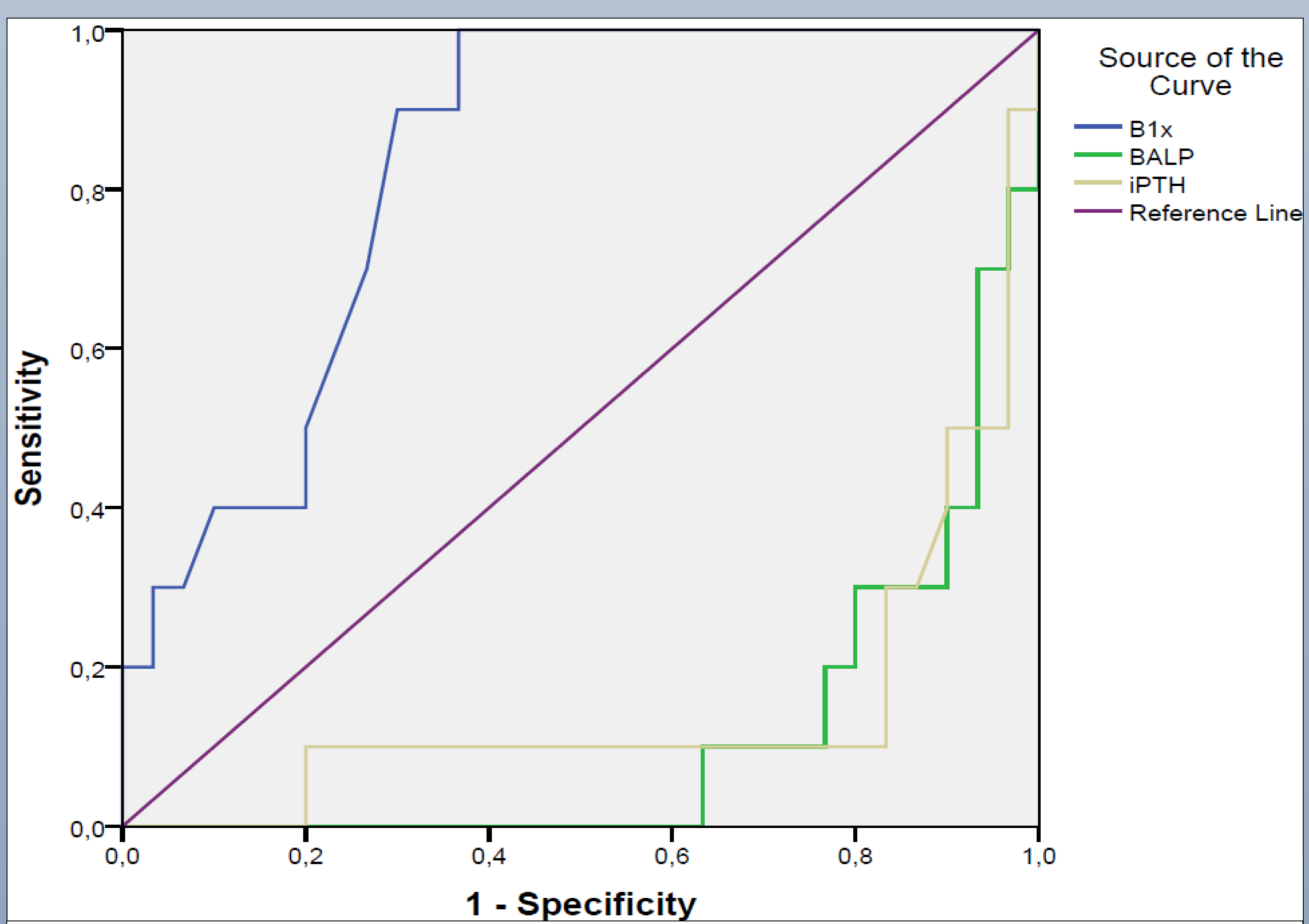

Receiver operator characteristic (ROC) curve for diagnosis of low mineral apposition rate (MAR)

AUC: B1X $0.828(p<0.01)$, BALP $0.113(p<0.001$, and PTH $0.148(p=0.001)$.

\begin{tabular}{|c|c|c|c|c|c|c|}
\hline \multicolumn{7}{|c|}{$\begin{array}{l}\text { Correlation coefficients of markers of bone metabolism and } \\
\text { histomorphometric parameters }\end{array}$} \\
\hline & N.Ob/B.Pm & Ob.Vg & BFR/BS & MAR & MIt & MS/BS \\
\hline$B / I$ & 0.18 & 0.12 & 0.08 & 0.03 & -0.10 & 0.09 \\
\hline B1x & -0.30 * & -0.26 * & -0.26 * & $-0.29 *$ & 0.28 * & $-0.25 *$ \\
\hline B1 & 0.19 & 0.16 & 0.16 & 0.14 & -0.15 & 0.17 \\
\hline B2 & 0.28 * & 0.19 & 0.20 & 0.16 & -0.18 & 0.20 \\
\hline BALP & $0.43^{* \star *}$ & 0.38 ** & 0.39 ** & $0.36^{* \star}$ & $-0.37^{\star *}$ & $0.38^{* *}$ \\
\hline PTH & $0.40^{* \star \star}$ & $0.39 * *$ & 0.37 ** & $0.33^{* *}$ & $-0.35^{* *}$ & $0.37^{* *}$ \\
\hline \multicolumn{7}{|c|}{$\begin{array}{l}\text { BALP, bone specific alkaline phosphatase; B/l, B1x, B1, B2, isoforms of BALP; PTH, } \\
\text { parathyroid hormone; N.Ob/B.Pm, number of osteoblast per bone perimeter; Ob.Vg; } \\
\text { osteoblast vigor; BFR/BS, bone formation rater per bone surface; MAR, mineral } \\
\text { apposition rate; MS/BS, mineralizing surface per bone surface; BFR/Ob, bone formation } \\
\text { rate per osteoblast; Mlt, mineralization lag time. }{ }^{*}=\mathrm{P}<0.05,{ }^{* *}=\mathrm{P}<0.01,{ }^{* * *}=\mathrm{P}<0.001\end{array}$} \\
\hline
\end{tabular}

